\title{
PERFIL DO CONSUMIDOR DE OVOS DE GALINHA NO MUNICÍPIO DE
} AQUIDAUANA-MS

\author{
Danilo Souza Sanches ${ }^{1}$ \\ Elis Regina de Moraes Garcia ${ }^{2}$ \\ Gislaine da Cunha de Andrade ${ }^{1}$ \\ Laura Ramos de Ávila ${ }^{1}$
}

\begin{abstract}
RESUMO
Objetivou-se avaliar o perfil dos consumidores de ovos do município de Aquidauana-MS. A pesquisa foi realizada por meio de entrevistas com consumidores diretamente nos postos de venda, localizados em diferentes pontos da cidade mediante a aplicação de um questionário previamente definido. Verificou-se que a grande maioria dos entrevistados era do sexo feminino (72,5\%), com idade entre 21 a 40 anos (47,5\%), com grau de instrução referente ao ensino superior completo $(28 \%)$ e renda familiar próxima a três salários mínimos $(50,5 \%)$. A maioria dos entrevistados consome ovos três vezes por semana, preferencialmente fritos (44\%) e avaliam a validade do produto na hora da compra $(66,8 \%)$. A maior parte da população entrevistada prefere ovos de casca vermelha $(66,5 \%)$, embora não ache que a cor da casca afete o seu valor nutricional $(58,9 \%)$. Ainda que 58\% dos consumidores entrevistados tenham afirmado que a cor da gema influencia o valor nutricional do ovo, $59,3 \%$ não tem preferência por essa característica. Observou-se percentual significativo (71\%) de consumidores que não possuem conhecimento do valor nutricional, mas que afirmam que o consumo de ovos não faz mal à saúde $(67 \%)$. O consumidor de ovos do município de Aquidauana - MS é representado em sua maioria por mulheres com idade entre 20 a 40 anos, com escolaridade superior completa, renda familiar ao redor de três salários mínimos e uma frequência de consumo de três vezes na semana. São exigentes no momento da compra, preferem ovos de casca vermelha, não se importam com a coloração da gema e acreditam que o ovo não faz mal à saúde, porém declaram que o alimento apresenta alta concentração de colesterol e que pode transmitir alguma doença como a salmonelose.
\end{abstract}

Palavra-Chave: alimentação, avicultura, consumo de ovos, pontos de venda, questionário.

\section{CONSUMER PROFILE OF CHICKEN EGGS IN THE MUNICIPALITY OF AQUIDAUANA, MS}

\begin{abstract}
The objective of this study was to evaluate the consumer profile of chicken egg in the municipality of Aquidauana-MS. The research was conducted through interviews with consumers directly at the Supermarkets, located in different parts of the city by applying a previously defined questionnaire. It was found that the majority of interviewed were female $(72,5 \%)$, aged between $21-40$ yaers $(47,5 \%)$, with an education degree referring to complete higher education $(28 \%)$ and family income close to three minimum wages $(50,5 \%)$. The most interviewed consumed eggs three times a week, preferably fried $(44 \%)$ and evaluate the validity of the product at the time of purchase $(66,8 \%)$. Most of the interviewed population chose shell eggs $(66,5 \%)$, although they do not think that the color of the shell affects its nutritional value $(58,9 \%)$. Even if $58 \%$ interviewed have stated that the color of the yolk

\footnotetext{
${ }^{1}$ Mestrando da Universidade Estadual do Mato Grosso do Sul. Correspondência: danilorzt9@gmail.com

${ }^{2}$ Professora Adjunta Universidade Estadual do Mato Grosso do Sul.
} 
influences the nutritional value of the egg $59,3 \%$ do not have prefer by this characteristic. It was observed significant percentage $(71 \%)$ of consumers who don't have knowledge of the nutritional value but who claim that egg consumption is not bad for health $(67 \%)$. The egg consumer of the municipality of Aquidauana - MS is mostly represented by women aged between 20 and 40 years, with complete higher education, family income close to three minimum wages and a frequency of consumption of three times a week. They are demanding at the time of purchase, shell eggs, do not care about the yolk color and believe that the egg is not bad for health, but declare that the food has a high concentration of cholesterol and that it can transmit some disease.

Keywords: food, poultry farming, egg consumption, points of sale, questionnaire.

\section{PERFIL DE LOS CONSUMIDORES DE HUEVOS DEL MUNICIPIO DE AQUIDAUNA-MS.}

\section{RESUMEN}

El objetivo fue evaluar el perfil de los consumidores de huevos del municipio de AquidaunaMS. La investigación fue realizada por medio de entrevistas con consumidores directamente en los puntos de venta, distribuidos en diferentes puntos de la ciudad, mediante cuestionarios definidos previamente. Se verificó que la mayoría de los entrevistados eran de sexo femenino (72,5\%), con edades de 21 a 40 años $(47,5 \%)$, con educación superior completa (28\%) e ingresos económicos familiares próximos a tres salarios mínimos $(50,5 \%)$. La mayoría de los entrevistados come huevo tres veces por semana, preferencialmente fritos (44\%) y evalúan la calidad del producto en el momento de la compra (66,8\%). La mayor parte de los entrevistados prefieren huevos de cáscara roja $(66,5 \%)$, sin embargo, no consideran que el color afecte su valor nutricional (58,9\%). Aunque el 58\% de los entrevistados afirmaron que el color de la yema influencia el valor nutricional del huevo, 59,3\% no tienen preferencia por esta característica. Se observó un porcentaje significativo $(71 \%)$ de consumidores que no poseen conocimiento del valor nutricional, pero afirman que el consumo de huevos no es perjudicial para la salud (67\%). El consumidor de huevos del municipio de Aquidauana - MS está representado principalmente por mujeres de entre 20 y 40 años de edad, con educación superior completa, ingresos familiares cercanos a tres salarios mínimos y una frecuencia de consumo de tres veces a la semana. Son exigentes en el momento de la compra, prefieren los huevos de cáscara roja, no se preocupan por el color de la yema y creen que el huevo no es malo para la salud, pero declarar que el alimento tiene una alta concentración de colesterol y que puede transmitir alguna enfermedad.

Palabras clave: alimentos, avicultura, consumo de huevos, puntos de venta, cuestionario

\section{INTRODUÇÃO}

Nos últimos anos, a cadeia produtiva da avicultura de postura vem se mostrando uma das atividades mais complexas e dinâmicas do segmento, tornando-a um pilar essencial para a economia brasileira.

Devido ao baixo custo e fácil acesso, os ovos são consumidos em maior quantidade, principalmente em países em desenvolvimento, com faixas de renda mais baixas (1). Além disso, é uma alternativa acessível para substituir a carne bovina e, em função do seu elevado valor nutricional (2) este produto é classificado como um alimento extremamente importante para alimentação humana, e seu consumo deve ser estimulado (3). 
O mercado consumidor vem sofrendo mudanças quanto as orientações sobre alimentação e saúde, assim, os consumidores a cada dia tornam-se mais exigentes com o produto adquirido, estando mais atentos sobre a qualidade, aspectos sensoriais, higienização, embalagem e presença de injurias, estão dispostos a adquirir e a pagar mais por produtos que apresentam tais características (4).

A percepção de compra dos consumidores está relacionada a aspectos regionais e culturais distintos e isso é respaldado de acordo com o tipo de comprador e produto. Segundo Mattar (5), a melhor técnica para medir a atitude e conhecer as necessidades, desejos e características do consumidor é o auto relato, o qual pode ser obtido por meio de entrevistas pessoais.

Assim, o conhecimento das preferências e comportamento dos consumidores quanto ao produto adquirido, principalmente sobre o preço e qualidade são importantes para que os produtores e a agroindústria possam reajustar componentes da cadeia produtiva que possibilitem melhorar a qualidade e, consequentemente, a comercialização dos ovos para consumo.

Deste modo, objetivou-se com o presente estudo identificar o perfil do consumidor de ovos no município de Aquidauana-MS.

\section{MATERIAL E MÉTODOS}

O referencial teórico que ampara a metodologia de estudo utilizado foi fundamentado na teoria do consumidor com as hipóteses neoclássicas da concorrência perfeita. Mais especificamente, utilizou-se a ideia de demanda (ou procura) do consumidor e os fatores que as influenciam, conforme Williamson (6).

O estudo foi desenvolvido no município de Aquidauana - MS, localizado na região noroeste do Estado de Mato Grosso do Sul a $130 \mathrm{~km}$ da capital, Campo Grande. De acordo com o Instituto Brasileiro de Geografia e Estatística a população estimada do município para o ano de 2019 é de 47.871 habitantes (7).

Adotou-se o método "survey" que é amplamente usada em pesquisas de opinião pública, de mercado e em pesquisas sociais. Essa metodologia é fundamentada por meio de entrevista, em que perguntas relacionadas ao tema estudado são direcionadas aos participantes por meio da aplicação de um questionário estruturado para obter uma padronização do processo de coleta de dados (8).

A pesquisa foi realizada em duas etapas. A primeira consistiu na elaboração e estruturação dos questionários, seguida da interação com os responsáveis dos locais de comercialização para a autorização da pesquisa. A segunda etapa compreendeu a realização de entrevistas com consumidores diretamente nos postos de venda, com a utilização dos questionários previamente definidos.

Foram selecionados os principais estabelecimentos que comercializam ovos no município localizados em diferentes pontos da cidade, o que proporcionou a obtenção da percepção de consumidores de diferentes classes sociais. Os entrevistados foram abordados de forma aleatória no interior dos mercados.

Para calcular o número de pessoas entrevistadas foi utilizado o método de amostragem sem reposição para uma população finita de acordo com Pinheiro e Lirio (9), em que a equação possui nível de confiança de $99 \%$ e tolerância de erro amostral de $1 \%$. Equação: $\mathrm{n}=$ (Z2.p.q.N) / [d2. (N-1) + Z2.p.q], em que $\mathrm{N}$ representa o tamanho da população; $\mathrm{Z}$ é a abscissa da curva normal padrão $(2,576)$; $\mathrm{p}$ é a estimativa da verdadeira proporção de um dos níveis da variável escolhida (no caso, $\mathrm{p}=\mathrm{q}=0,5$ ) e d é o erro amostral admitido.

Foram avaliados o gênero, idade, renda familiar, nível de formação, frequência e forma de consumo de ovos, critérios considerados na escolha do produto, preferência de cor de casca 
e gema, conhecimento sobre o valor nutricional, benefícios para saúde humana, certificação do produto (inspeção), consumo de ovos do tipo caipira e conhecimento sobre o processo produtivo e se os consumidores tinham conhecimento se o ovo transmite alguma doença.

Com o intuito de aprimorar os questionários foi aplicado um pré-teste em $10 \%$ da população entrevistada, para que pudessem ser corrigidos eventuais erros de formulação das perguntas, obtendo-se assim uma resposta efetiva para as variáveis investigadas. No momento da aplicação dos questionários evitou-se induzir os entrevistados as respostas, utilizando perguntas curtas e objetivas e o entrevistado não tinha acesso visual às alternativas.

Os dados foram submetidos à analise descritiva mediante a técnica de determinação das frequências percentuais, observadas de acordo com as categorias das variáveis.

\section{RESULTADOS E DISCUSSÃO}

Constatou-se que a maioria dos consumidores de ovos de galinha pertence ao sexo feminino $(72,5 \%)$, de faixa etária de 21 a 40 anos $(47,5 \%)$, com ensino superior completo (28\%) e renda familiar próxima a três salários mínimos $(50,5 \%)$. Tais valores afirmam que as mulheres, por serem as responsáveis pelo lar e mais organizadas financeiramente, tendem a ser encarregar pelas compras e alimentação na maioria dos lares da região.

Dados do Instituto Brasileiro de Geografia e Estatística (IBGE), revelam que o país possui mais mulheres entre 25 a 44 anos com ensino superior completo e que realizam suas atividades domésticas totalmente sozinhas e que elas são as responsáveis pelas compras e alimentação de $96 \%$ dos domicílios brasileiros, ainda que essas exerçam atividade remunerada (10).

No que se refere a frequência de consumo observou-se que a maioria dos entrevistados consome ovos três vezes por semana (Figura 1). Tal resultado é considerado satisfatório, já que este valor está dentro da média nacional (212 ovos/hab/ano), de acordo com dados da Associação Brasileira de Proteína Animal (11).

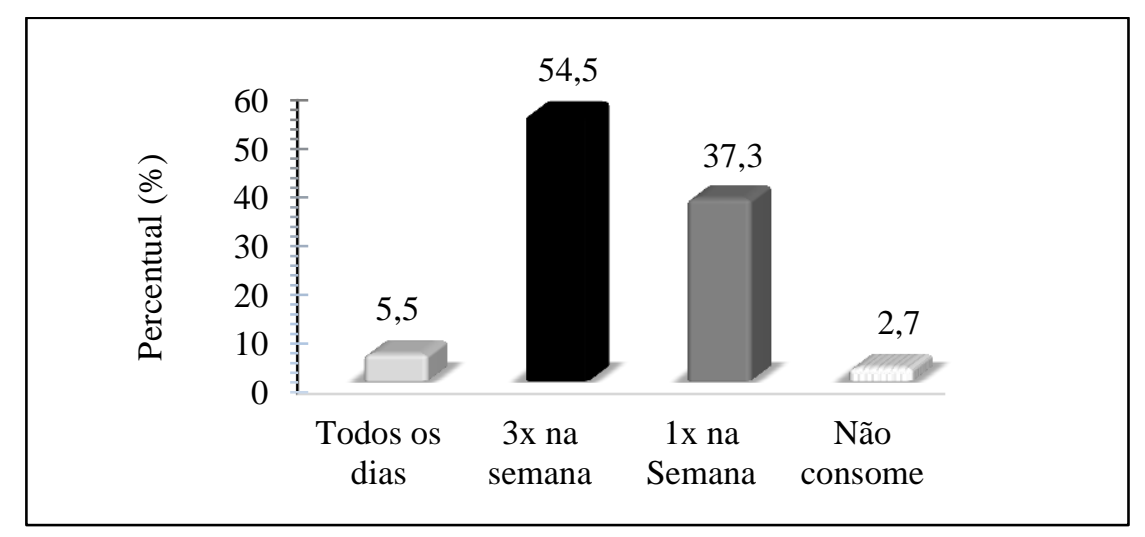

Figura 1. Frequência de consumo de ovos de poedeiras comerciais pelos consumidores do município de Aquidauana- MS

Em relação a frequência de consumo, os resultados obtidos no presente trabalho se assemelham aos encontrados por Silva et al. (12) no Nordeste que, ao investigarem a frequência de consumo de ovos de galinha na região de Teresina- PI, observaram que 33\% dos entrevistados consomem ovos três vezes por semana. Da mesma forma, Vivas et al. (13), estudando o perfil do consumidor de ovos no município de Ilha Solteira-SP, afirmaram que $38 \%$ dos entrevistados consomem ovos apenas uma vez na semana. 
De acordo com os dados encontrados no presente trabalho, o elevado consumo de ovos pode estar relacionado com a baixa renda familiar da população do município, recorrendo assim, a produtos de preço mais acessível.

Em relação à forma de consumo, $44 \%$ dos entrevistados consomem ovos fritos, $28 \%$ por meio da elaboração de outros pratos e $28 \%$ preferem os ovos cozidos. Tal resultado confirma a falta de conhecimento da população sobre o preparo do alimento, já que preferem consumir ovos fritos e não cozidos, que é a forma de preparo mais saudável (14).

Uma vez que o consumidor se torna a cada dia mais exigente em relação a qualidade e formas de acesso ao alimento que consomem, pode-se observar que os consumidores da região são bastante criteriosos no momento da escolha do produto, pois 49,5\% dos entrevistados afirmaram que todos os aspectos questionados como preço, aspecto do ovo, embalagem, higiene e procedência são levados em consideração no momento da compra (Figura 2).

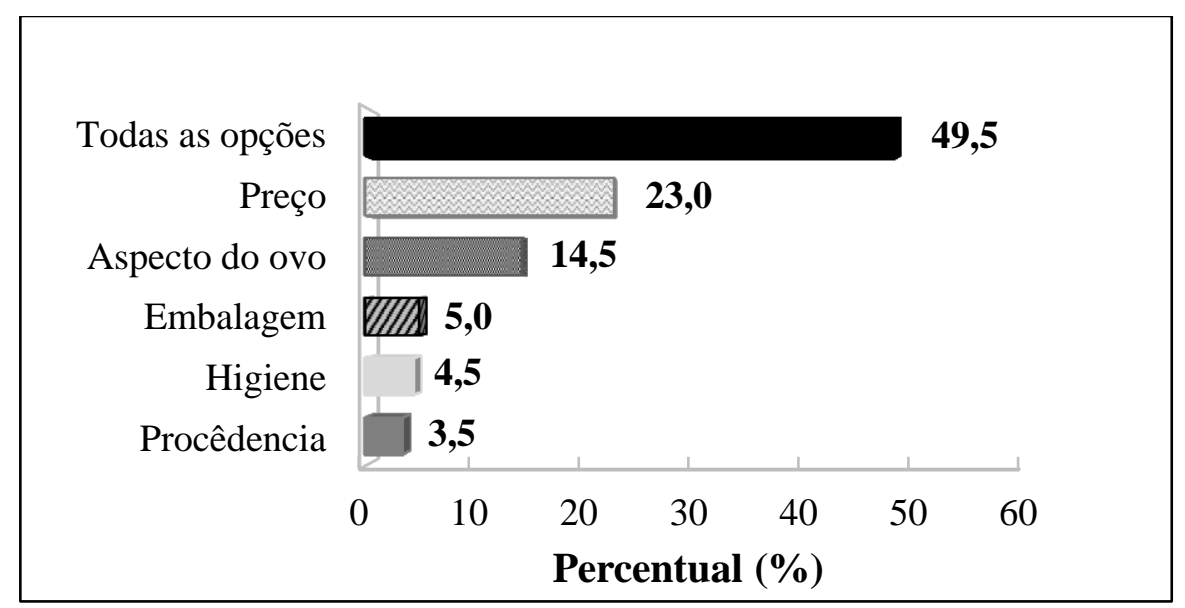

Figura 2. Fatores que influenciam a compra de ovos pelos consumidores do município de Aquidauana- MS.

Silva et al. (12) e Fernandes et al. (15) identificaram que para a maioria dos consumidores entrevistados do município de Teresina-PI e Machado- MG, respectivamente, o preço do produto foi mais importante, demonstrando que o valor econômico é mais relevante que a qualidade do produto nessas regiões. Por outro lado, na cidade de Ilha Solteira- SP, Vivas et al. (13) observaram que o critério de escolha de ovos pelos consumidores é a procedência do produto. Esses estudos diferem do presente trabalho, pois os aquidauanenses mostraram ser mais preocupados e exigentes quanto ao produto adquirido.

Os dados coletados demonstraram que $66,8 \%$ dos entrevistados conferem a validade do produto no momento da compra, no entanto, há um valor expressivo $(33,2 \%)$ de consumidores que não se preocupam com essa informação. Outro aspecto relevante, é que $51,5 \%$ dos entrevistados não possuem conhecimento sobre o serviço de inspeção municipal, federal ou estadual na venda dos ovos para consumo, demonstrando novamente a falta de preocupação dos consumidores ao adquirir os ovos.

Quanto a validade do produto, conforme a legislação vigente no país, o decreto $\mathrm{n}^{\mathrm{o}}$ 56.585 de 20 de julho de 1965 respalda que é obrigatório conter no rótulo das embalagens as informações básicas como: tipo de ovo, classe, grupo, data de classificação, prazo de validade e identificação do lote (16). Como todos os produtos naturais de origem animal, o ovo também é perecível e começa a perder seu valor nutritivo após a postura, caso não sejam tomadas medidas adequadas para sua conservação. Segundo Freitas et al. (17), em 
temperatura ambiente, a validade máxima de um ovo sem deteriorar a qualidade interna varia de quatro a quinze dias após a data de postura.

Em relação ao conhecimento dos consumidores sobre os aspectos físicos e valor nutricional do ovo, 66,5\% dos entrevistados não possuem preferência pelo tamanho e preferem ovos de casca vermelha, embora 58,9\% declararam que a cor da casca não influencia o valor nutricional do ovo. Apesar da maioria afirmar que não existe diferença nutricional entre ovos de casca vermelha e branca, considera-se alto o percentual de entrevistados $(41,1 \%)$ que ainda assegura que essa diferença existe, sendo uma percepção incorreta. Do ponto de vista nutricional, Araújo e Albino (18) relatam que ovos de coloração de casca diferente apresentam valores nutricionais equivalentes, ou seja, ambos são ricos em proteínas, vitaminas, sais minerais e possuem cerca de 220 miligramas de colesterol.

É evidente a falta de conhecimento por parte dos entrevistados sobre os fatores genéticos atribuídos as linhagens de poedeiras que produzem ovos de coloração de casca distintas, uma vez que, a capacidade que a ave possui em depositar pigmentos denominados porfirina na casca, são regulados e depositados por vários genes que atuam nas glândulas calcíferas localizadas no útero do animal, é o que define a coloração da casca do ovo (19).

Verificou-se que um número significativo de entrevistados (71\%) não possui conhecimento sobre o valor nutricional do ovo, mas $52 \%$ afirmam que o mesmo é um alimento nutricionalmente completo e que pode substituir outro tipo de alimento (60\%), como a carne. A opinião da população sobre a complexidade do valor nutricional do produto é correta, pois o ovo possui um perfil de micronutrientes essenciais que são incomparáveis com qualquer outro alimento, além de possuir uma proteína de elevado valor biológico superior ao da carne e semelhante ao do leite (20).

Quando questionados sobre a coloração da gema, 59,3\% dos entrevistados afirmaram que não possuem preferência, no entanto, $58 \%$ afirmam que a cor da gema influencia o valor nutricional do ovo. A intensidade de cor da gema é uma variável de qualidade que influência a escolha do produto pelo consumidor (21), para os brasileiros a gema deve ser altamente pigmentada, ou seja, maior que oito no abanico de cores da DSM (22), pois há uma associação entre a cor da gema e a sua quantidade de vitaminas (23).

Divergindo dos resultados deste estudo, trabalhos realizados em Natal-Rio Grande do Norte (24), Angelim, Garanhuns, Lagoa do Ouro, São João - Pernambuco (25) e TeresinaPiauí (12), com o objetivo de analisar a preferência dos consumidores de ovos, demonstraram que na região do nordeste há maior preferência por ovos de casca branca. Segundo os autores, esses dados são interessantes, devido a maior frequência de compradores que acreditam no mito de que ovos de casca vermelha são mais nutritivos.

Este resultado demonstrou que a preferência por ovos da casca branca nessas regiões pode ser mais atribuído a questão econômica do que pela falta de conhecimento dos consumidores, já que os ovos brancos tem menor custo que os vermelhos.

A maioria dos entrevistados consome ovo caipira (68,5\%), atribuindo sua preferência ao fato desses ovos apresentarem melhor sabor, em função do sistema de criação, maior valor nutricional e intensidade da cor da gema (Figura 4). Esse resultado corrobora com a preferência dos consumidores por ovos vermelhos no presente trabalho, demonstrando a presença de um nicho de mercado para este tipo de produto na região, já que os aquidauanenses demonstraram um interesse satisfatório por esse tipo de ovos.

Quando os entrevistados foram questionados sobre a relação entre o consumo de ovos e saúde humana (Figura 3), a maior parte dos consumidores relatou que o ovo não faz mal à saúde, mas que possui elevada concentração de colesterol, ao passo que afirmam que o produto pode transmitir algum tipo de doença, mencionando a bactéria Salmonella como a principal. Tais resultados podem ser explicados pelo nível de escolaridade dos entrevistados, pois a maioria possui ensino superior completo, atribuindo assim, um conhecimento mais 
elevado sobre os assuntos questionados. Segundo Griffin (26), grande parte da população mundial ainda apresenta restrições em relação ao consumo de ovos, devido ao equívoco desatualizado de um "mito", de que o colesterol do ovo é ruim e que pode causar doenças cardiovasculares.

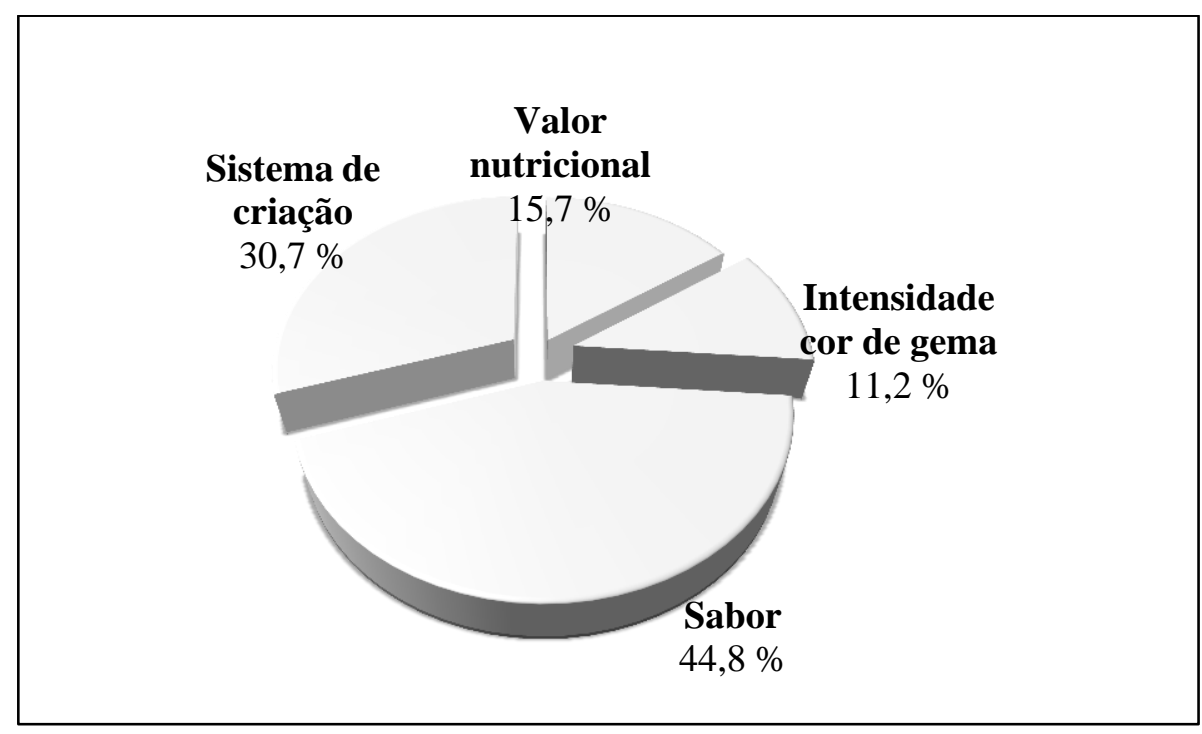

Figura 3. Fatores que influenciam a preferência dos consumidores por ovos do tipo caipira

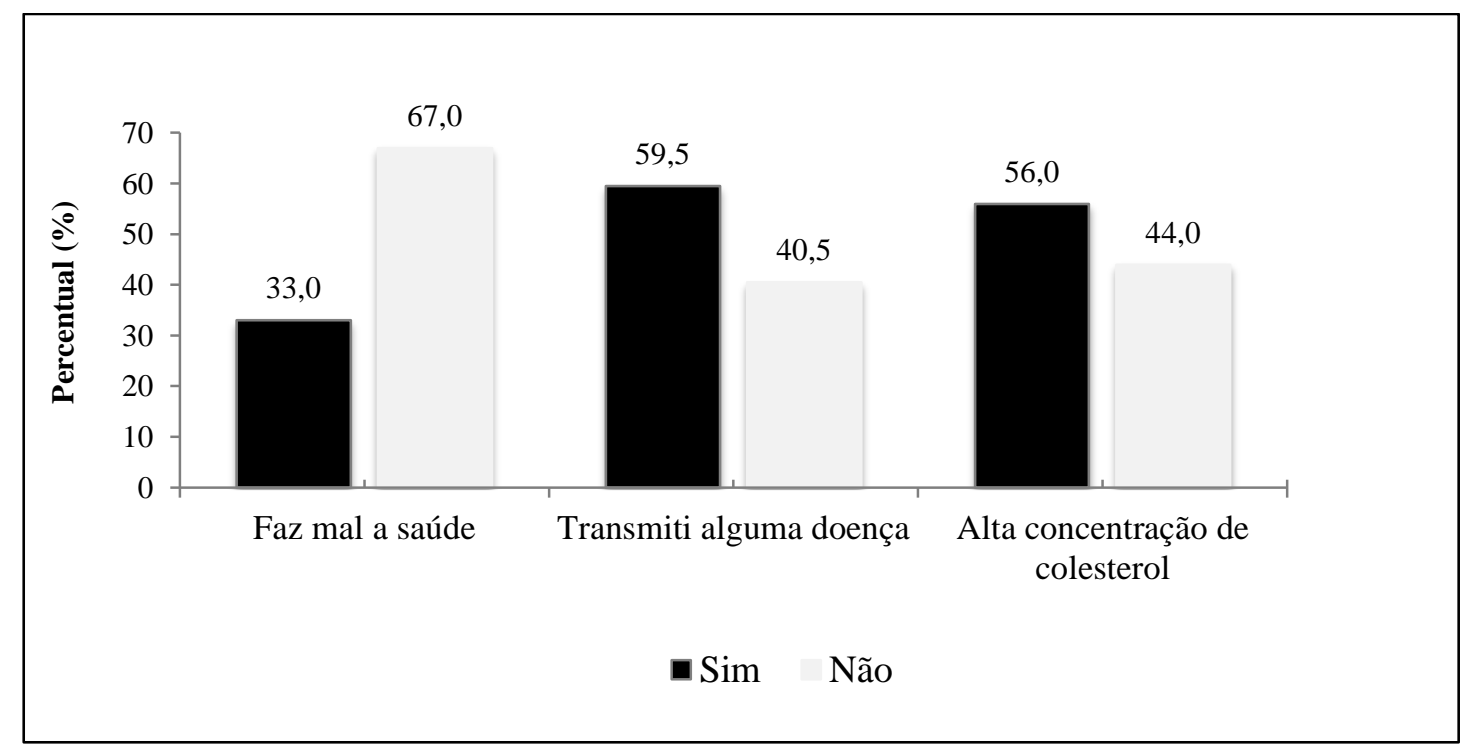

Figura 4. Conhecimento do consumidor sobre a relação entre o consumo de ovos e saúde humana

O volume crescente de evidências adquiridas por meio de meta-análises (27) e revisões sistemáticas (28) respaldam que o colesterol alimentar oriundo de ovos exerce efeito relativamente pequeno e clinicamente insignificativo sobre o colesterol sérico comparado a outros fatores, como o estilo de vida das pessoas. Barraj et al. (29) concluíram que a mudança do estilo de vida (má alimentação, tabagismo, sedentarismo e ingestão de álcool) foi responsável por reduzir em $40 \%$ a mortalidade por doença cardíaca de indivíduos com idade acima de 25 anos, e o consumo de um ovo por dia contribuiu apenas com $1 \%$. 
É evidente a falta de conhecimento da população sobre assuntos básicos referente ao ovo, assim a divulgação e esclarecimento dessas por meio dos rótulos das embalagens dos ovos, marketing criativos nos estabelecimentos de venda e criação de páginas virtuais que possibilitem um maior conhecimento a respeito do produto é essencial.

\section{CONCLUSÕES}

O consumidor de ovos do município de Aquidauana - MS é representado em sua maioria por mulheres com idade entre 20 a 40 anos de idade, com escolaridade superior completa, renda familiar ao redor de três salários mínimos e uma frequência de consumo elevada, três vezes na semana. São consumidores exigentes no momento da compra, verificando a validade do produto, embora não possuam conhecimento sobre o serviço de inspeção sanitária. Além disso, preferem ovos de casca vermelha, não se importam com a coloração da gema e acreditam que o ovo não faz mal à saúde, porém declaram que o alimento apresenta alta concentração de colesterol e que pode transmitir alguma doença, como a salmonelose.

\section{REFERÊNCIAS}

1. Stefanello C. Análise do sistema agroindustrial de ovos comerciais. Rev Agrar. 2011;4(14):375-82.

2. Oliveira C. Alimentação. Ovo: consumo com segurança. Lavoura. 2012;115(691):54 -7.

3. Donato DCZ, Gandra ÉRS, Garcia PDSR, Reis CBM, Gameiro AH. A questão da qualidade no sistema agroindustrial do ovo. In: Anais da 47o Congresso da Sociedade Brasileira de Economia, Administração e Sociologia Rural; 2009; Porto Alegre (RS). Porto Alegre: Sober; 2009.

4. Moura AMA, Oliveira NTE, Thiebaut JTL, Melo TV. Efeito da temperatura de estocagem e do tipo de embalagem sobe a qualidade interna de ovos de codorna japonesas (Coturnix japonica). Cienc Agrotec. 2008;32(2):578-83.

5. Mattar FN. Pesquisa de marketing. São Paulo: Atlas; 1996. (Edição completa).

6. Williamson OE. The new institutional economics: taking stock, looking ahead. J Econ Lit. 2000;38(3):595-613.

7. Instituto Brasileiro de Geografia e Estatística. Censo demográfico 2010 [Internet]. Rio de Janeiro: IBGE; 2010 [cited 2020 Mar 25]. Available from: http://www.ibge.gov.br/home/estatistica/populacao/censo2010/resultados_dou/MS2010.p df

8. Malhotra N. Pesquisa de marketing: uma orientação aplicada. 3a ed. Porto Alegre: Bookman; 2001.

9. Pinheiro CD, Lírio VS. Fatores condicionantes da demanda de carne suína no município de Viçosa-MG. Rev Econ UMA. 2003;8(2):43-66. 
10. Instituto Brasileiro de Geografia e Estatística. Estatística de gênero: indicadores sociais das mulheres no Brasil. Rio de Janeiro: IBGE; 2018. (Estudos e Pesquisas Informação Demográfica e Socioeconômica n. 38).

11. Associação Brasileira de Proteína Animal. Relatório anual: produção Brasileira [Internet]. São Paulo: ABPA; 2019 [cited 2020 Mar 28]. Available from: http://abpabr.org/mercados/\#relatorios

12. Silva MB, Raposo JDAS, Ramos LSN. Consumidores de ovos de galinha do município de Teresina, PI. Rev Bras Pesqui Aliment. 2015;6(1):56-63.

13. Vivas DN, Pantolfi N, Diniz RF, Silva Junior CD, Rubio MS, Laurentiz AC. Perfil do consumidor de ovos de poedeiras comerciais no município de Ilha Solteira-SP [Internet]. In: Anais do 7o Encontro de Ciências da Vida; 2013; Ilha Solteira. Ilha Solteira: ENCIVI; 2013 [cited 2020 Mar 28]. Available from: http://www.feis.unesp.br/Home/Eventos/encivi/viiencivi-2013/36---perfil-doconsumidor-de-ovos-de-poedeiras-comerciais-no-municipio-de-ilha-solteira---sp.pdf

14. Novello D, Quintiliano PFDA, Ost PR. Ovo: conceitos, análises e controvérsias na saúde humana. Arch Latinoam Nutr. 2006;56(4):315-20.

15. Fernandes MR, Souza AV, Maciel MLS, Morais MVM, Pimenta GF, Pereira LGB, et al. Perfil do consumidor de ovos de poedeiras comerciais no município de Machado-MG. In: Anais do Zootecnia Brasil; 2018; Goiânia (GO). Goiânia: PUC-GO; 2019.

16. Brasil. Decreto 56.585, de 20 Julho 1965. Aprova as novas especificações para a classificação e fiscalização do ovo. Diário Oficial da União. 22 Jul 1965; Sec. 1, p. 6954.

17. Freitas LW, Paz ICLA, Garcia RG, Caldara FR, Seno LO, Felix GA, et al. Aspectos qualitativos de ovos comerciais submetidos a diferentes condições de armazenamento. Rev Agrar. 2011;4(11):66-72.

18. Araujo WAG, Albino LFT. Comercial incubation. Trivandrum, Kerala, IND: Transworld Research Network; 2011.

19. Oliveira HF, Carvalho DP, Ismar MG, Rezende PM, Camargo SMP, Souto CN, et al. Fatores intrínsecos a poedeiras comerciais que afetam a qualidade físico-química dos ovos. Pubvet. 2020;14(3):1-11.

20. Benelam B, Roe M, Pinchen H, Church S. New data on the composition of UK hens' eggs. Nutr Bull. 2012;37(4):344-9.

21. Ziggers D. Astaxantina: un corante y ademas saludable. Avicultura Profesional. 2000;20(8):12-3.

22. Bertechini AG. Nutrição de monogástricos. 2a ed. Lavras: Editora UFLA; 2012.

23. Garcia EA, Mendes AA, Pizzolante CC, Gonçalves HC, Oliveira RP, Silva MA. Efeito dos níveis de cantaxantina na dieta sobre o desempenho e qualidade dos ovos de poedeiras comerciais. Rev Bras Cienc Avic. 2002;4(1):1-7. 
24. Lopes EC, Oliveira CRC, Chung S, Luz WAS, Araújo ÍIM, Souza JG. Perfil do Consumidor de ovos de galinha na cidade de Natal - RN. In: Anais do 11o Congresso Internacional de Zootecnia; 2009; Águas de Lindóia (SP). Águas de Lindóia: SBZ; 2009. p.1-3.

25. Nunes EAC, Patrício PFMM, Patrício LAMM, Andrade ARS, Brasil KQ. Análise estatística da qualidade de ovos vermelhos, comercializados nos município de Angelim, Garanhuns, Lagoa do Ouro e São João - PE. In: Anais da 9o Jornada de Ensino, Pesquisa e Extensão - JEPEX; 2009; Recife-PE. Recife: Universidade Federal Rural de Pernambuco; 2009.

26. Griffin BA. Eggs: good or bad. Proc Nutr Soc. 2016;75(3):259-64.

27. Rong Y, Chen L, Zhu T, Canção Y, Yu M, Shan Z, et al. Egg consumption and risk of coronary heart disease and stroke: dose-response meta-analysis of prospective cohort studies. BMJ. 2013;346:e8539.

28. Tran NL, Barraj LM, Heilman JM, Scrafford CG. Egg consumption and cardiovascular disease among diabetic individuals: a systematic review of the literature. Diabetes Metab Syndr Obes. 2014;7:121-37.

29. Barraj L, Tran N, Mink P. A comparison of egg consumption with other modifiable coronary heart disease lifestyle risk factors: a relative risk apportionment study. Risk Anal. 2009;29(3):401-15. 